\title{
GRAVITATIONAL REDSHIFT OF EMISSION LINES IN THE AGN SPECTRA
}

\author{
Nataša Bon $^{1}$, Edi Bon ${ }^{1}$, Paola Marziani ${ }^{2}$ and \\ Predrag Jovanović ${ }^{1}$
}

\begin{abstract}
The detection of gravitationally redshifted optical emission lines has been reported just for a few active galaxies. In this paper we give a short overview of studies that analyzed or exploited the detection of the gravitational redshift in optical AGN spectra. In addition, we tested the consistency of gravitational redshift as the physical origin of the redward shifts observed in their spectra using a sample of $\approx 50$ Hamburg-ESO intermediate to high redshift quasars that are among the most luminous quasars known $\left(10^{47} \lesssim L \lesssim 10^{48} \mathrm{erg} / \mathrm{s}\right)$, and are expected to host very massive black holes. To this aim we modeled the line profile with accretion disk models around a black hole.
\end{abstract}

Keywords galaxies: active ; galaxies: emission lines ; techniques: spectroscopic ; Line: profiles ; Galaxies: Seyfert ; Accretion, accretion disks ; Gravitation

\section{Introduction}

A large fraction of active galactic nuclei show red asymmetries in their broad optical emission lines. The asymmetry is strong in Balmer lines, but even stronger redward asymmetry is displayed in the lines that originate closer to the central black hole (BH) such as HeII $\lambda 4686$, HeI $\lambda 5876$ or CIV $\lambda 1549$ (Peterson \& Wandel 1999; Peterson 2001; Kollatschny 2003). One of the proposed explanation of the red asymmetry is that the broad emission line gas is gravitationally dominated by the central black hole. In this regard, following the dichotomy of quasars proposed by Sulentic et al. (2000) onto Pop. A and Pop B. according to their

Nataša Bon, Edi Bon, Paola Marziani and Predrag Jovanović

1 Astronomical Observatory, Belgrade, Serbia

2 INAF, Padova, Italy spectral properties, a high fraction of Pop. B objects that are associated to AGN with the most massive BHs (Zamfir et al. 2010) show redshifted profiles. Corbin (1995) gave the quantitative test if the gravitational redshift ( $\mathrm{z}_{\text {grav }}$ ) model is possible, by calculating the mass of the central source, and found that the mass corresponding to the shift $z \sim 0.001$ should yield to the mass of around $10^{9} M_{\odot}$. This mass is close to the largest masses estimated for quasar black hole, since they rarely exceed $5 \cdot 10^{9} M_{\odot}$, and are almost always $\lesssim 10^{10} M_{\odot}$ (Czernv et al. 2004; Sulentic et al. 2006; Marziani \& Sulentic 2012).

In this paper we give a brief overview on previous gravitational redshift analyses. Also, we consider the origin of the redshift in the sample of $\approx 50$ Hamburg-ESO (HE) intermediate to high redshift quasars, that are among the most luminous quasars known $\left(10^{47} \lesssim L \lesssim 10^{48} \mathrm{erg} / \mathrm{s}\right)$, and are expected to host very massive black holes. We then matched simulated relativistic emission line profiles obtained by ray tracing method with the optical emission $\mathrm{H} \beta$ line profiles from HE sample, for the cases where centroids of line widths measured on $1 / 4$ of maximum line intensity were significantly shifted to the red, in order to see if their shapes could be distorted by gravitational redshift.

The paper is organized as follows. In Section 2 an overview on the gravitational redshift hypothesis is presented. Implications of gravitational redshift in the case of weak gravitational field are analyzed in Section 3. In Section 4 we consider the possibility that the gravitational redshift shapes the red wing of $\mathrm{H} \beta$ profile. Section 5 gives our conclusions on the gravitational redshift search in optical AGN spectra. 
2 Overview: AGN emission lines and gravitational redshift

The concept that gravitational redshift may influence quasar optical emission lines causing line asymmetries was proposed by Netzer (1977) 1 There were some earlier attempts to consider gravitational redshift as an explanation for the cosmological redshift of quasars but this led to a series of inconsistencies (see Greenstein \& Schmidt 1964). The systematic gravitational redshift may have a non-negligible effect on the shape of spectral lines, when the emission region is in the gravitational field of a massive black hole $\left(M \sim 10^{7}-10^{10} M_{\odot}\right)$ (Netzer 1977; Anderson 1981; Popović et al. 1995; Corbin 1997). This leads to an asymmetric and redshifted profile of emission lines, especially in broad lines (BL) or their very broad line (VBL) base that originate at distances of only few light days from the central black hole (Popović et al. 1995).

Peterson et al. (1985) suggested gravitational redshift as an explanation for the red shifted very broad wings in the optical emission line profiles in order to explain the redward asymmetric broad $\mathrm{H} \beta$ profile of Arakelian 120, under the assumption of very compact broad line region (BLR). This effect was more systematically investigated by Sulentic (1989) and Zheng \& Sulentic (1990) who assumed a single component model of the broad line region, and by Corbin (1995) who assumed a two component model, a very broad line region (VBLR, where profile bases are formed) and an intermediate line region (ILR, forming the profile cores) for the BLR. Corbin (1997) studied relativistic effects on the emission from the BLR assuming orbits for the emitting clouds in a Schwarzschild geometry, and proposed a model for the BLR in which he distinguished two emitting regions, the ILR and the VBLR, taking into account the relativistic effects on the emission line profiles. In that paper he suggested that redward profile asymmetries can be produced by the effect of gravitational redshift on the emission from the VBLR, when the VBLR is represented by a flattened ensemble of clouds viewed nearly face-on, at a mean distance of few tens of gravitational radii from the black hole. The redshift of the ILR component could be as well explained with the same geometry of a flattened ensemble of clouds lying on a distance of several hundreds gravitational radii from the central black hole.

\footnotetext{
${ }^{1}$ In the following line shifts will be considered with respect to the quasar rest frame i.e., after removing the effect of cosmological redshift.
}

Corbin 1993, 1995) found that the red asymmetry of $\mathrm{H} \beta$ line increases with the increasing soft X-ray luminosity. This correlation may be interpreted as a progressive redshift of the very broad line region velocity centroid ( 5000-20000 $\mathrm{km} \mathrm{s}^{-1}$ ) with respect to the systemic velocity with increasing X-ray luminosity. Also, Corbin (1995) noticed that, on average, radio-loud (RL) objects show stronger redward asymmetric profiles than radio-quiet (RQ) object of comparable optical luminosity. Under the gravitational redshift hypothesis, these differences could be explained as the larger masses of the black hole and the host galaxy for RL objects. This is consistent with the evidence that they are merger products (Schweizer 1982; Kormendy \& Diorgovski 1989; Heckman et al. 1986; Smith et al. 1986; Hutchings 1987; Wilson \& Colbert 1995). However, this interpretation may not be correct because RQ in the same black hole mass domain are also found (Marziani et al. 2003; Zamfir et al. 2010).

Since the variable part of the spectral line originates far more closely to the central nuclei than the constant line components, Peterson \& Wandel (1999); Kollatschny \& Dietrich (1997) and Kollatschny (2003) assumed that the gravitational redshift effect might be present in this variable component. Therefore, they used the rms profile of the spectra from the monitoring campaign of NGC5548 (Peterson \& Wandel 1999), NGC4593 (Kollatschny \& Dietrich 1997) and Mrk 110 (Kollatschny 2003), measured the shift between the rms profile and the mean profile, and identified the differential shift as gravitational redshift. Kollatschny (2003) analyzed the shift in four emission lines $\mathrm{H} \alpha, \mathrm{H} \beta$, HeI $\lambda 5876$ and HeII $\lambda 4686$ and found the strongest $z_{\text {grav }}$ in the $\operatorname{HeII} \lambda 4686$, as it originates closest to the black hole. The confirmation that the optical emission lines in Mrk 110 are gravitationally affected was given by Müller \& Wold (2006), where emission lines are modeled under the assumption that they originate in the vicinity of a black hole.

\subsection{Exploitation of gravitational redshift}

If line redshift with respect to rest frame and redward line asymmetries are indeed of gravitational origin, it would be possible to derive parameters that are important in studies of AGN spectra, such as:

- the SMBH mass, with an orientation-independent estimate - Knowing the gravitational redshift $z_{\text {grav }}$, one can estimate an upper limit of the central mass as $M_{\text {grav }} \sim c^{2} z_{\text {grav }} r G^{-1}$ (Zheng \& Sulentid 1990). This way of estimating the SMBH mass is independent of the geometry and inclination of the object, 
but still requires the knowledge of the emitting region radius $(\mathrm{r})$. Other methods for mass estimations that assume the virial motion of the gas, such as $M_{\text {orbital }}=f v^{2} G^{-1} r$ (Wandel et al. 1999), include the dimensionless factor $f$ that depends, among other things, on the geometry and orientation of the BLR.

- the inclination angle of the accretion disk - in the case of Mrk 110, Kollatschny (2003) assumed the accretion disk model of the BLR. Under this assumption, the comparison between $M_{\text {grav }}$ and $M_{\text {orbital }}$ give an estimation of the orientation angle $i$ of the accretion disk : $M_{\text {orbital }} / M_{\text {grav }}=\sin ^{2} i$. Therefore, this method makes it possible to estimate the inclination angle of the accretion disk for radio-quiet AGNs. Until now, the inclination angle has been estimated only for a relatively small number of radioloud sources in which superluminal motion has been detected (Sulentic et al. 2003; Rokaki et al. 2003). Recently, (Piotrovich et al. 2015) presented the new method for the estimations of the inclination of the broad emission line region (BLR), based on the observations in a polarised light, under the assumption of a flattened configuration of BLR, but this interesting method has been tentatively applied until now only to a few tens of Seyfert nuclei.

- Spin orientation of the supermassive black hole Kollatschnv (2003) went one step further in the interpretation of the inclination angle. From the crosscorrelation analysis he determined the distances of the line emitting regions, using the rms profile. He found that the variable HeII $\lambda 4686$ line component originates at a mean distance of 3.9 light days, that mean 230 Schwarzschild radii only. Therefore, he suggested that the orientation axis of the inner accretion disk is oriented parallel to the spin axis of the central black hole.

However, the nature of the systematic line shift still remains an open question, since shifts in both directions - redward, but also blueward are observed. Redshifts of $\mathrm{H} \beta$ are observed more frequently than blueshifts, and that could indicate a systematic trend that may not be immediately explainable by Doppler effect assuming plausible kinematic scenarios. Collin et al. (2006) analysed symmetric aspect of $\mathrm{H} \beta$ line and concluded that $\mathrm{H} \beta$ line profile is primarily sensitive to Eddington ratio, but that inclination effects play role in some cases, such as narrow line Seyfert 1 galaxies (NLS1). The fraction of redshifted profiles is very high in Pop. B, usually associated to AGN with the most massive BHs (Zamfir et al.2010). Beside gravitational redshift, both radial and transverse velocity could account for the observed term. Among them, the gravitational term could
Table 1 Table shows the difference of the gravitational red shift contribution calculated using Schwarzschild and weak field approximation.

\begin{tabular}{llll}
\hline $\begin{array}{l}\text { Radius } \\
\left(\mathrm{R}_{\mathrm{S}}\right)\end{array}$ & $\begin{array}{l}\mathrm{Z}_{\text {grav }} \\
\text { Schwarzschild }\end{array}$ & $\begin{array}{l}\mathrm{Z}_{\text {grav }} \\
\text { Weak field }\end{array}$ & Diff. \\
\hline 20 & 0.025978 & 0.025000 & $4 \%$ \\
30 & 0.017095 & 0.016667 & $3 \%$ \\
40 & 0.012739 & 0.012500 & $2 \%$ \\
100 & 0.005038 & 0.005000 & $1 \%$ \\
200 & 0.002509 & 0.002500 & $0 \%$ \\
\hline
\end{tabular}

be the most probable cause, since the transverse term is on the same order of magnitude as the gravitational one, and in fact implies a dominant gravitational field (Zheng \& Sulentic 1990).

\section{The test for the redshift origin of optical emission lines in AGN sample - is it really gravitational redshift?}

A sample of $\approx 50$ Hamburg-ESO (HE) intermediate to high redshift quasars have been observed in the $\mathrm{H} \beta$ spectral range using the IR spectrometer ISAAC (Marziani et al. 2009, M09, and references therein). These sources are among the most luminous quasars known $\left(10^{47} \lesssim L \lesssim 10^{48} \mathrm{erg} / \mathrm{s}\right)$, and are expected to host very massive black holes, as indicated by the large $\mathrm{H} \beta$ FWHM: all black hole masses computed from the scaling relation of Vestergaard and Peterson (2006) are indeed $\log M>9$. The Population B part of this sample has a high prevalence of redshifted centroid:2: $\approx$ $80 \%$ of all Pop. B sources show a significant centroid displacement to the red at $1 / 4$ fractional intensity (Fig. 9 of M09). The $\mathrm{H} \beta$ redward asymmetry is well correlated with the black hole mass (Fig. 16 of M09).

Therefore, these luminous sources represent an appropriate sample to test the consistency of gravitational redshift as the physical origin of the redward shifts observed in Pop. B. Under the assumption of weak field in the Schwarzschild metric, one can write for the combined effect of gravitational plus transverse redshift

$\Delta z \approx \frac{3}{2} \frac{G M}{R c^{2}}=\frac{3}{4} \frac{R_{\mathrm{S}}}{R}$

If $\mathcal{C}$ indicates the line centroid, then $\Delta z=\frac{\mathcal{C}}{c}$, and $R=\frac{3 c}{4} \mathcal{C}^{-1} R_{\mathrm{S}}$, or $R \approx 225 \cdot \mathcal{C}_{1000}^{-1} \quad R_{\mathrm{S}}$, where the centroid is now in units of $1000 \mathrm{~km} \mathrm{~s}^{-1}$, and $R_{\mathrm{S}}$ is the

${ }^{2}$ Centroid is calculated as follows: $\mathcal{C}\left(\frac{\mathrm{i}}{4}\right)=\frac{\lambda_{\mathrm{B}}\left(\frac{\mathrm{i}}{4}\right)+\lambda_{\mathrm{R}}\left(\frac{\mathrm{i}}{4}\right)}{2}-\lambda_{0}$, for $\mathrm{i}=1,3$, where $\lambda_{R}$, and $\lambda_{B}$, represent the wavelength on the red and blue side, respectively, measured at $i / 4$ level of the line intensity. 
Schwarzschild radius. Basically a centroid displacement of $1000 \mathrm{~km} \mathrm{~s}^{-1}$ corresponds to a distance from the central mass of about $200 R_{\mathrm{S}}$. We can consider the centroid at $1 / 4$ intensity $\mathcal{C}\left(\frac{1}{4}\right)$, or after a decomposition of the $\mathrm{H} \beta$ profile into a broad component (BC) and a very broad component (VBC), the peak of the VBC. For the $\mathrm{HE}$ sample, the median value of the VBC peak shifts is $\approx 2200 \mathrm{~km} \mathrm{~s}^{-1}$, and $\mathcal{C}\left(\frac{1}{4}\right) \approx 1600 \mathrm{~km} \mathrm{~s}^{-1}$, corresponding to just $\sim 100$ Schwarzschild radii (note that even at about $30 R_{S}$ the weak field formula differs by only $3 \%$ from the correct Schwarzschild expression, see Table 1).

In Table 2 we report several parameters for a sample of $16 \mathrm{HE}$ quasars (out of 28 of the sample of Sulentic et al., in preparation) showing an $\mathrm{H} \beta$ redward asymmetry. The Columns of Table 2 list in the following order - bolometric luminosity, black hole mass, $\mathcal{C}\left(\frac{1}{4}\right)$, VBC peak shift, VBC FWHM, fraction of flux in the VBC, the VBC luminosity, as well as the distances needed for explaining the observed redward displacement due to gravitational+transverse redshift, as derived from the $\mathcal{C}\left(\frac{1}{4}\right)$ and from the VBC shift. From the median mass $\log M \approx 9.65$, we derive median distances of 140 (from $\mathcal{C}\left(\frac{1}{4}\right)$ ) and $100 R_{S}$ (from VBC shift), which correspond to linear sizes of $\sim 1.8$ and $1.4 \cdot 10^{17} \mathrm{~cm}$ (see Table 2). This radius may be considered as an emissivity weighted radius, and certainly not the inner radius of the disk.

Here we try to answer the question: Is there enough gas close to the central black hole that is able to emit the very luminous redshifted emission components observed in the HE quasars? A first hint, at the expected $\mathrm{H} \beta$ luminosity, may come from the assumption of a photoionized medium in case B. Under this assumption the covering factor of the emitting gas should be less than 1 (typically $0.1-0.2$, no more than 0.5 ) for gravitational redshift to produce a consistent result. A low covering factor is possible only for high density $\left(\gtrsim 10^{11} \mathrm{~cm}^{-3}\right.$ ) and large column density $\left(\sim 10^{25} \mathrm{~cm}^{-2}\right)$. However, at such high $L$ and small radii, a case $B$ photoionization may not be possible at the illuminated face of the cloud. The temperature is too high and the gas remains fully ionized even very deep in a slab of dense gas. An explorative CLOUDY simulation (Ferland et al. 1998) assuming median values from Table 2 for luminosity $\left(\log L_{b o l} \approx 47.5\right)$, radius $(\log r \approx 17.2)$, high density and column density $(\log n=12, \log N=14$, respectively), indicates that the gas is able to emit just $\sim 1 / 50$ of the observed VBC luminosity, with full covering. The total VBC emission predicted by CLOUDY of $\mathrm{H} \beta$ is about 2 order of magnitudes lower than that predicted from simple case B considerations. The amount of gas at the small distances from the $\mathrm{BH}$ derived from the assumption of gravitational redshift is not favored as a significant source of $\mathrm{H} \beta$ emission for the very luminous HE sources: there is simply not enough gas in the available volume of radius $\sim 100 \cdot R_{S}$. This conclusion could well be of general validity, since VBC shifts of $\approx 2000$ $\mathrm{km} \mathrm{s}^{-1}$ are observed also in low-luminosity sources.

It is not obvious where some cold gas can survive so close to the central black hole, at less than $\approx 100$ Schwarzschild radii. In the case of an optically thin advection-dominated accretion flow (ADAF, see e.g. Narayan et al. 1996, 1997), the electron temperature should be of the order of the virial temperature. The gas temperature at $100 R_{S}$ from a $10^{9} M_{\odot}$ black hole is $T_{e} \sim 4 \cdot 10^{6} \mathrm{~K}$, that is too high. On the other hand, the photospheric temperature of an geometrically thin, optically thick accretion disk at $100 R_{S}$ for the same mass is $\sim 2 \cdot 10^{3} \dot{M}_{26}^{1 / 4} \mathrm{~K}$. The disk is directly illuminated by only $\sim 5 \cdot 10^{-4}$ of the total quasar $L$ at 100 $R_{S}$. Continuum radiation could be also scattered toward the disk (i.e., by an atmosphere of hot electrons). This case requires an evaluation of the heating effects of the continuum radiation over the vertical structure of the disk itself, and may be worth a new detailed study, along the line followed by Dumont et al. (1990).

\section{Could relativistic line profile describe distorted AGN emission line profiles?}

In some AGN, emission lines are detected in X-ray spectral region, like Fe $\mathrm{K} \alpha$ spectral line (6.4-6.9 keV, depending on ionization state). It is expected that for high masses ranging from $10^{5}$ to $10^{9}$ solar masses $\mathrm{M}_{\odot}$, the innermost accretion disk that surrounds central super-massive black hole should radiate in the $\mathrm{X}$ ray band (Fabian et al. 1989). Fluorescent / recombination iron $\mathrm{K} \alpha$ emission line has been observed in a number of AGN (see e.g. Nandra et al. 2007; Yaqoob et al. 2001; Yaqoob \& Padmanabhan 2004; Page et al. 2004; Shu et al. 2010). It is thought to be produced by the hard X-ray power law continuum from the hot corona around central $\mathrm{BH}$ which irradiate relatively cold inner part of accretion disk (Fabian et al. 2000). Therefore, it is expected to be very broad (velocities $\approx 50000$ $\mathrm{km} \mathrm{s}^{-1}$, Nandra et al. 1997) and asymmetric due to special relativistic effects and large rotational velocities of material emitting near the black hole, showing very narrow "blue" peak and wider and much fainter "red" peak (Fabian et al. 2000). General relativistic effects can further distort the line shapes, so the red wing becomes more extended towards lower energies for higher values of the BH spin (see Revnolds \& Nowak 2003; Jovanović \& Popović 2008; Jovanović et al. 2011; Jovanović 2012), while the line becomes wider and its red peak brighter. 
Table 2 Measured quantities for the HE sample: bolometric luminosity, black hole mass, $\mathcal{C}\left(\frac{1}{4}\right)$, VBC peak shift, VBC FWHM, fraction of flux in the VBC, the VBC luminosity, as well as the distances needed for explaining the observed redward displacement due to gravitational+transverse redshift, as derived from the $\mathcal{C}\left(\frac{1}{4}\right)$ and from the VBC shift.

\begin{tabular}{|c|c|c|c|c|c|c|c|c|c|c|}
\hline $\begin{array}{l}\text { Name } \\
\mathrm{erg} / \mathrm{s}\end{array}$ & $\begin{array}{c}L_{b o l}\left(f_{\lambda}\right) \\
M_{\odot}\end{array}$ & $M_{V P 06}$ & $\begin{array}{l}\mathrm{L} / L_{E d d} \\
\mathrm{~km} \mathrm{~s}^{-1}\end{array}$ & $\begin{array}{r}\mathrm{c}(1 / 4) \\
\mathrm{km} \mathrm{s}^{-1}\end{array}$ & $\begin{array}{c}\text { VBC shift } \\
\mathrm{km} \mathrm{s}^{-1}\end{array}$ & $\begin{array}{c}\text { FWHM VBC } \\
\mathrm{km} \mathrm{s}^{-1}\end{array}$ & $\mathrm{VBC} /$ tot & $\begin{array}{c}\mathrm{L}(H \beta) \mathrm{VBC} \\
\mathrm{erg} / \mathrm{s}\end{array}$ & $\begin{array}{c}d_{C} \\
\left(R_{S}\right)\end{array}$ & $\begin{array}{c}d_{V B C} \\
R_{S}\end{array}$ \\
\hline HE0035-2853 & 47.37 & 9.74 & -0.582 & 1880 & 5470 & 11240 & 0.39 & $1.38 \mathrm{E}+45$ & 119.3 & 41.1 \\
\hline HE0058-3231 & 47.31 & 9.51 & -0.417 & 2500 & 3350 & 9150 & 0.59 & $2.31 \mathrm{E}+45$ & 90.0 & 67.0 \\
\hline HE0203-4627 & 47.33 & 9.70 & -0.787 & 1040 & 2060 & 12390 & 0.42 & $7.70 \mathrm{E}+44$ & 217.1 & 109.1 \\
\hline HE0205-3756 & 47.94 & 9.57 & -0.108 & 2620 & 2270 & 12360 & 0.50 & $5.20 \mathrm{E}+45$ & 85.9 & 99.0 \\
\hline HE0251-5550 & 47.97 & 9.61 & 0.128 & 2890 & 2060 & 11310 & 0.59 & $5.87 \mathrm{E}+45$ & 77.8 & 109.0 \\
\hline HE0349-5249 & 47.72 & 9.73 & -0.238 & 2200 & 2380 & 11250 & 0.69 & $4.66 \mathrm{E}+45$ & 102.4 & 94.5 \\
\hline HE0436-3709 & 47.20 & 9.56 & -0.475 & 2050 & 2140 & 11190 & 0.49 & $1.35 \mathrm{E}+45$ & 109.6 & 105.3 \\
\hline HE0926-0201 & 47.75 & 9.62 & -0.113 & 800 & 950 & 13010 & 0.56 & $3.74 \mathrm{E}+45$ & 281.1 & 237.1 \\
\hline HE1039-0724 & 47.27 & 10.00 & -0.942 & 1940 & 2430 & 12170 & 0.35 & $1.13 \mathrm{E}+45$ & 115.6 & 92.5 \\
\hline HE1120+0154 & 47.66 & 9.83 & -0.434 & 1270 & 2400 & 14040 & 0.53 & $3.24 \mathrm{E}+45$ & 177.4 & 93.6 \\
\hline HE1349+0007 & 47.21 & 9.52 & -0.404 & 1410 & 3110 & 13070 & 0.44 & $1.18 \mathrm{E}+45$ & 160.0 & 72.3 \\
\hline HE1409+0101 & 47.77 & 10.03 & -0.796 & 1590 & 2230 & 14560 & 0.54 & $6.16 \mathrm{E}+45$ & 141.4 & 100.7 \\
\hline HE2156-4020 & 47.69 & 9.57 & -0.152 & 1620 & 1440 & 13050 & 0.60 & $5.96 \mathrm{E}+45$ & 138.9 & 156.7 \\
\hline HE2202-2557 & 47.28 & 9.65 & -0.434 & 900 & 3000 & 12340 & 0.48 & $9.63 \mathrm{E}+44$ & 249.8 & 74.9 \\
\hline HE2349-3800 & 47.37 & 9.45 & -0.512 & 1100 & 1450 & 11090 & 0.66 & $1.88 \mathrm{E}+45$ & 204.4 & 154.9 \\
\hline HE2355-4621 & 47.71 & 9.85 & -0.413 & 1500 & 2150 & 13900 & 0.39 & $4.03 \mathrm{E}+45$ & 149.9 & 104.6 \\
\hline
\end{tabular}
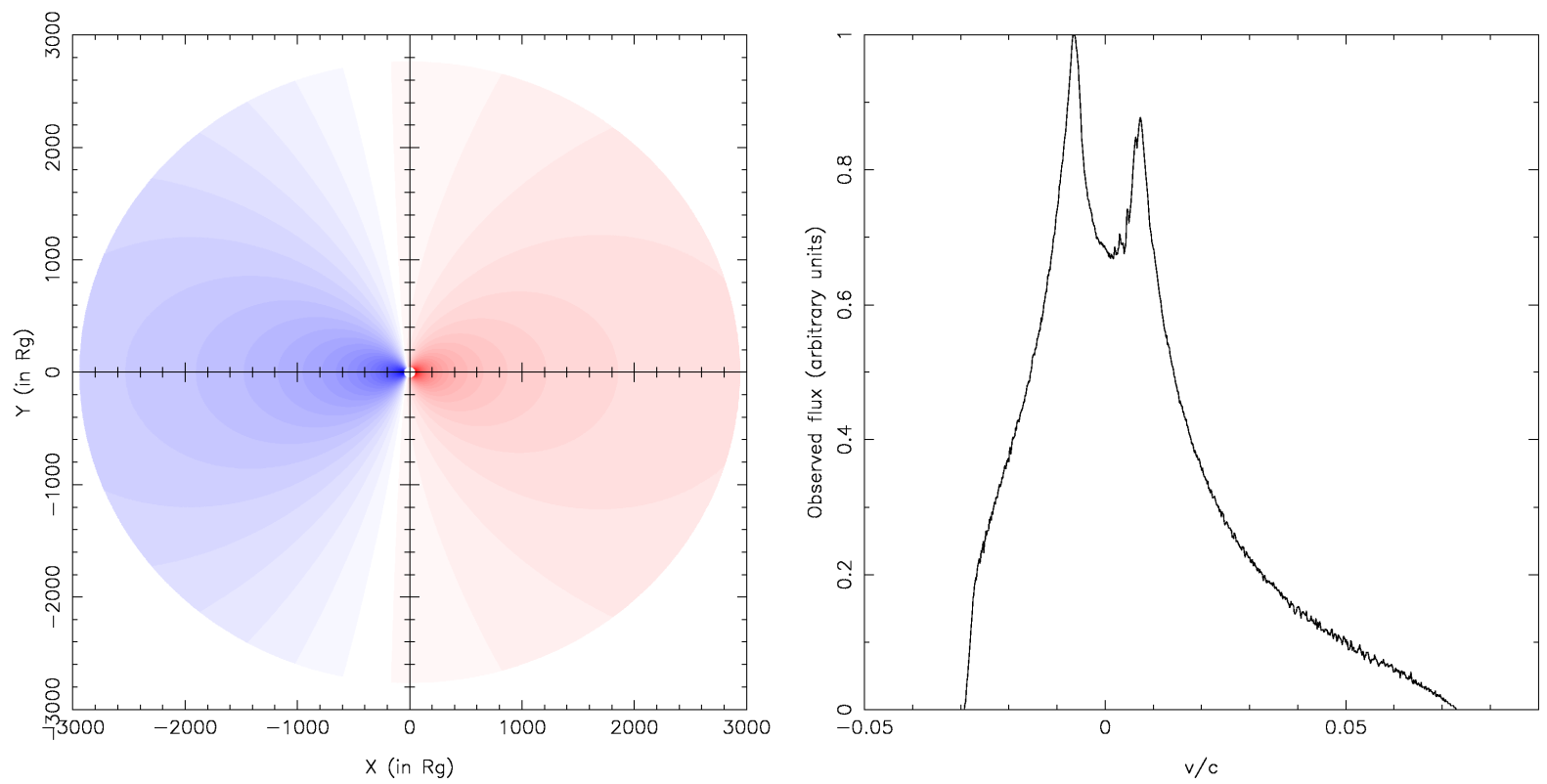

Fig. 1 Example of an image of a simulated relativistic accretion disk (left panel) and corresponding profile of $\mathrm{H} \beta$ line (right panel), for inclination $i=20^{\circ}$, emissivity index of 2.2 and radii $\operatorname{Rinn}=50 \mathrm{Rg}$ and $\operatorname{Rout}=4000 \mathrm{Rg}($ where $\mathrm{Rg}=\mathrm{Rs} / 2)$, obtained by ray tracing method in Kerr metric (Jovanovió 2012). 

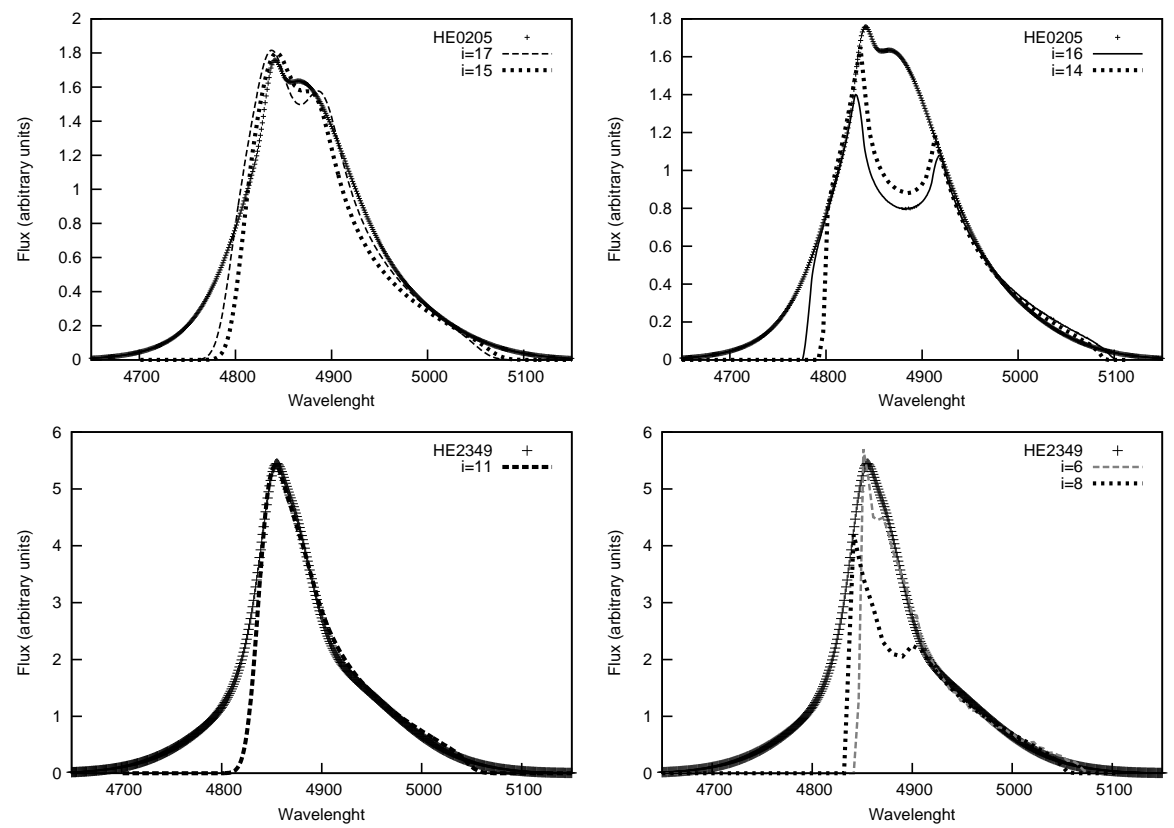

Fig. 2 Examples of $\mathrm{H} \beta$ profiles compared with relativistic disk profiles calculated using very small inner radius (Rinn < $80 \mathrm{Rg})$. On the top panels is the broad component of the $\mathrm{H} \beta$ emission line of the object HE0205, while on the bottom panels are broad emission lines of HE2349. On the left hand side emission lines are compared with the Chen \& Halpern (1989) disk model, while on the right hand panels they are compared with the Kerr relativistic disk model, obtained by ray tracing method (see e.g. Čadež et al. 1998; Jovanović 2012). The inclinations are presented on the panels. The other parameters are presented in the Table 3

In comparison to Fe K $\alpha$ emission line, optical emission lines, such as $\mathrm{H} \beta$ line, are expected to be emitted from more distant region then $\mathrm{Fe} \mathrm{K} \alpha$, and therefore the gravitational redshift effect in $\mathrm{H} \beta$ is expected to be much weaker.

We simulated relativistic emission line profiles by using ray tracing relativistic method (Čadež et al. 1998; Jovanović 2012; J Jovanović et al. 2011; Jovanović \& Popovi 2008; Müller \& Wold 2006), assuming that emitting regions are thin equatorial rings in stationary Keplerian rotation. We simulated the $H \beta$ line profiles using the code which includes both special relativistic (transverse Doppler shift and Doppler beaming), general relativistic (gravitational redshift) effects on radiation from the accretion disk around SMBH (for more details see e.g. Jovanović \& Popović 2009; Jovanović 2012, and references therein), and which is based on ray-tracing method in the Kerr metric (Fanton et al. 1997; Čadež et al. 1998). Although this code was developed for studying the X-ray radiation from innermost regions of the disk, it can be also applied for simulating the optical emission from its outer regions (see e.g. Jovanović et al. 2010).

The example of a simulated accretion disk image and the corresponding $\mathrm{H} \beta$ line profile are presented in the Figure 10
For the optical emission lines, most commonly used model of the relativistic accretion disk is model of Chen \& Halpern (1989). It was developed to explain mainly double peaked profile of optical emission lines, therefore it was mainly tested on complex emission line profiles of Seyfert type 1.5 AGN (like 3c390.3 and Arp 102B). There were some attempts where Then \& Halpern (1989) relativistic disk model was used to explain single peaked broad emission line shapes (Popović et al. 2004; Bon et al. 2006, 2009a, b). Such simulations assumed the inner radius of the disk relatively far from the SMBH, starting from few hundred $\mathrm{Rg}$, where the effects of gravitational redshift were not significant.

Here we tried to simulate broad emission line profiles using Chen \& Halpern (1989) relativistic disk model, assuming that the inner radius of the disk should originate very close to the SMBH (with values less than hundred gravitational radii), where the gravitational redshift effect is significant, with the aim to explain the shape of $H \beta$ extended red wing with gravitational effects.

Simulated profiles are compared to broad, $\mathrm{H} \beta$ optical emission lines from the HE sample, for the cases where centroids of line widths measured on $1 / 4$ of maximum line intensity were significantly shifted to the red. 
Table 3 Table lists parameters (inclination $(i)$, power law emissivity index $(e)$, inner and outer radius, given in gravitational radii (Rinn, and Rout), respectively) of the Chen \& Halpern (1989) disk model (CH89), and the ray tracing relativistic disk model (see e.g. Jovanovic 2012, (J12)), used to match $\mathrm{H} \beta$ emission line of two spectra from HE sample.

\begin{tabular}{llllll}
\hline Name & Disk model & $i$ & $q$ & Rinn $(\mathrm{Rg})$ & Rout $(\mathrm{Rg})$ \\
\hline HE0205 & CH89 & 17 & -2.22 & 80 & 8000 \\
HE0205 & CH89 & 15 & -2.2 & 65 & 12000 \\
HE0205 & J12 & 16 & -2.2 & 75 & 1100 \\
HE0205 & J12 & 14 & -2.2 & 70 & 1000 \\
HE2349 & CH89 & 11 & -2.5 & 58 & 15000 \\
HE2349 & J12 & 6 & -2.5 & 40 & 5000 \\
HE2349 & J12 & 8 & -2.6 & 53 & 500 \\
\hline
\end{tabular}

We matched the simulated line profiles with observed $\mathrm{H} \beta$ emission lines in order to see if we could find the solution which could match their distorted red wing, assuming the effects of gravitational redshift, considered in relativistic disk models. Müller \& Wold (2006) calculated that for the optical lines with resolution of $0.1 \AA$ gravitational redshift in the line profiles becomes important at distances smaller then 75000 gravitational radius $(\mathrm{Rg})$. Since the emissivity weighted radii calculated for HE sample (Table 2) are very close to the BH, we assumed that inner radius (Rinn) of the disk model should start from similar values. The emission lines are compared with the two relativistic accretion disk models: Chen \& Halpern (1989) model for relativistic disk in Schwarzschild metric, and accretion disk model in Kerr metric (see e.g. Čadež et al. 1998; Jovanović 2012). Starting from the values presented in Table 1 for the inner radius of the accretion disk, we searched for the shape that looked similar as the red side of the emission line profile, while changing the disk parameters. Examples of simulated disk profiles matched to the observed broad emission lines of HE0205 and HE2349 are represented in the Fig. 2] with corresponding parameters of each model presented in Table 3

As could be seen from Fig. 2, the red wing of the line profile could be well described with relativistic accretion disk model for relatively small range of face on inclinations and inner radius relatively close to the $\mathrm{BH}$. This is in agreement with results from Müller \& Wold (2006).

We note here that such model could not describe the line blue wing. Therefore, one would need to assume that, if the accretion disk contributes to some part of the line flux, it cannot explain the shape of the blue wing. Then at least part of the blue wing, and probably part of the line core needs to be emitted from some other, kinematically different region (outflows, winds, jets, etc.). Similar models were sugested earlier, assuming at least two kinematically different components (the disk with additional emitting component) relatively close to BH (see e.g. Corbin 1997; Popović et al. 2004; Bon et al. 2006, 2009a, b, 2012). Another difficulty is that the emissivity law $\left(\sim r^{q}\right)$ is very steep (with $q$ between -2.2 and -2.6), weighting emission in favor of the inner disk region, where gas may be too hot and it is not clear if it can significantly contribute to the total line emission.

If the red wing of the emission line originate in the accretion disk, then one could expect to observe in monitoring campaigns of some objects that the red wing respond to the continuum variations faster then the blue wing (see some cases where this is observed, e.g. Gaskell \& Goosmann 2013; Done \& Krolik 1996; Welsh et al. 2007; Gaskell 2009). Of course, there are many open questions about accretion disks (Sulentic et al. 1999), and many alternative models are suggested to explain the BLR model, like inflows, outflows, binary or multiple black holes (see e.g. Bogdanović 2015; Bon et al. 2012; Popović 2012), and many more.

\section{Summary}

It has been known since 20 years that redward asymmetry of very broad wings are common in the Type 1 Pop. B AGN (see e.g. Peterson et al. 1985; Marziani \& Sulentic 2012). The median $\mathrm{H} \beta$ profile of Pop. B type of AGN show such asymmetry (see e.g. Marziani \& Sulentic 2012). This phenomenon could be connected to the effects of gravitational redshift. Some other alternative explanation of redshifted emission line could be that the redshift is due to radial motion, either inflows (Gaskell \& Goosmann 2013) or outflows (see e.g. Zheng \& Sulentid 1990).

We found that the red wing of the line profile could be well described with the relativistic accretion disk models for relatively small range of face on inclinations and very close inner radii. This indicates that in some cases the optical emission lines could be distorted by relativistic effects and gravitational redshift.

However, it is not clear whether there is enough gas close to the central black hole that is able to emit the very luminous redshifted emission components that are observed in the HE quasars. Simple estimates suggest that this might be unlikely, probably because most gas is expected to be too hot to significantly emit Balmer lines, but a detailed model of an illuminated accretion disk is needed to properly estimate line emissivity. If there is indeed enough gas to sustain power law emissivity (with $q$ between $\approx-2.2$ and $\approx-2.6$ ), gravitational redshift could be estimated from the line centroid, and in this way $z_{\text {grav }}$ could be used as a proxy for $\mathrm{M}_{\text {grav }}$ calculation. 


\section{Acknowledgements}

This research is part of the projects 176001 "Astrophysical spectroscopy of extragalactic objects" and 176003

"Gravitation and the large scale structure of the Universe" supported by the Ministry of Education and Science of the Republic of Serbia. 


\section{References}

Anderson, K. S. 1981, ApJ, 246, 13

Bogdanović, T. 2015, Astrophysics and Space Science Proceedings, 40, 103

Bon, E., Jovanović, P., Marziani, P., Shapovalova, A. I., Bon, N., Borka Jovanović, V., Borka, D., Sulentic, J., Popović, L. č, 2012, ApJ, 759, 118

Bon, E., Popović, L. Č., Gavrilović, N., Mura, G. L., \& Mediavilla, E. 2009, Mon. Not. R. Astron. Soc., 400, 924

Bon, E., Gavrilović, N., La Mura, G., \& Popović, L. Č. 2009, New Astron. Rev., 53, 121B

Bon, E., Popović, L. Č., Ilić, D., \& Mediavilla, E. 2006, New Astron. Rev., 50, 716

Chen, K., \& Halpern, J. P. 1989, Astrophys. J., 344, 115

Collin, S., T. Kawaguchi, T., Peterson, B. M., Vestergaard M. 2006, Astron. Astrophys., 456, 75

Corbin, M., 1993, ApJ, 403, 9

Corbin, M., 1995, ApJ, 447, 496

Corbin, M., 1997, ApJ, 485, 522

Czerny, B., Różańska, A., Kuraszkiewicz, J. 2004, A\&A, 428,39

Čadež, A., Fanton, C., \& Calvani, M. 1998, New Astron., 3,647

Done, C., \& Krolik, J. H. 1996, Astrophys. J., 463, 144

Dumont, A. M., Collin-Souffrin, S. 1990, A\&A, 229, 313

Fabian, A. C., Rees, M. J., Stella, L., \& White, N. E. 1989, Mon. Not. R. Astron. Soc., 238, 729

Fabian, A. C., Iwasawa, K., Reynolds, C. S., \& Young, A. J. 2000, Publ. Astron. Soc. Pac., 112, 1145

Fanton, C., Calvani, M., de Felice, F., Cadez, A.: Detecting Accretion Disks in Active Galactic Nuclei. 1997, Publ. Astron. Soc. Jpn., 49, 159

Ferland, G. J., Korista, K. T., Verner, D. A., Ferguson, J. W., Kingdon, J. B., Verner, E. M. 1998, PASP, 110, 761

Gaskell, C. M. 2009, New Astron. Rev., 53, 140

Gaskell, C. M., \& Goosmann, R. W. 2013, ApJ 769, 30

Greenstein, J. L., \& Schmidt, M. 1964, ApJ, 140, 1

Heckman, T. M., Smith, Eric P., Baum, Stefi A., van Breugel, W. J. M., Miley, G. K., Illingworth, G. D., Bothun, G. D., Balick, B. 1986, ApJ, 311, 526

Hutchings, J. B. 1987, ApJ, 320, 122

Jovanović, P. 2012, New Astron. Rev., 56, 37

Jovanović, P., Borka Jovanović, V., \& Borka, D. 2011, Baltic Astronomy, 20, 468

Jovanović, P., Popović, L. Č., Stalevski, M., Shapovalova, A. I. 2010, ApJ, 718, 168

Jovanović, P., \& Popović, L. Č. 2008, Fortschritte der Physik, 56, 456

Jovanović, P., Popović, L. Č.: X-ray Emission From Accretion Disks of AGN: Signatures of Supermassive Black Holes, chapter in book "Black Holes and Galaxy Formation", Eds. A. D. Wachter and R. J. Propst, Nova Science Publishers, Inc, Hauppauge NY, USA, ISBN: 978-161761-398-2, 249 (2009), arXiv:0903.0978

Kollatschny, W. 2003, A\&A, 412, 61

Kollatschny, W. \& Dietrich, M. 1997, A\&A, 323, 5.

Kormendy, J. \& Djorgovski, S. 1989, ARAA, 27, 235

Marziani, P., Sulentic, J. W., Stirpe, G. M., Zamfir, S., Calvani, M. 2009, A\&A, 495, 83
Marziani, P., Sulentic, J. W., Zamanov, R., et al. 2003, Astrophys. J. Suppl. Ser., 145, 199

Marziani, P., \& Sulentic, J. W. 2012, New Astron. Rev., 56, 49

Müller, A. \& Wold, M. 2006, A\&A, 457, 485

Nandra, K., George, I. M., Mushotzky, R. F., Turner, T. J., Yaqoob, T. 1997, ApJ, 477, 602

Nandra, K., O'Neill, P. M., George, I. M., \& Reeves, J. N. 2007, Mon. Not. R. Astron. Soc., 382, 194

Narayan R., McClintock J.E.,Yi I. 1996, ApJ 457, 82

Narayan R., Barret D., McClintock J.E., 1997, ApJ, 482, 448

Netzer, H. 1977, MNRAS, 181, 89

Page, K. L., O’Brien, P. T., Reeves, J. N., \& Turner, M. J. L. 2004, Mon. Not. R. Astron. Soc., 347, 316

Peterson, B. M., Meyers, K. A., Capriotti, E. R., Foltz, C. B., Wilkes, B. J., \& Miller, A. R. 1985, ApJ, 292, 164

Peterson, B. M., \& Wandel, A. 1999, ApJ, 521, 95

Peterson, B.M 2001, Proceedings of a conference on The starburst-AGN connection, ed. I.Aretxaga, Singapore: World Scientific, p.3

Piotrovich, M. Y., Gnedin, Y. N., Silant'ev, N. A., Natsvlishvili, T. M., Buliga, S. D. 2015, arXiv:1509.01028

Popović, L. Č., Vince, I., Atanacković - Vukmanović, O., Kubičela, A. 1995, A\&A, 293, 309

Popović, L. Č., Mediavilla, E., Bon, E., \& Ilić, D. 2004, Astron. Astrophys., 423, 909

Popović, L, Č, 2012, New Astron. Rev., 56, 74.

Reynolds, C. S., \& Nowak, M. A. 2003, Phys. Rep., 377, 389

Rokaki, E., Lawrence, A., Economou, F., Mastichiadis, A. 2003, MNRAS, 340, 1298

Schweizer, F. 1982, ApJ, 252, 455

Shields, Joseph C., Ferland, Gary J., Peterson, Bradley M. 1995, ApJ, 441, 507

Shu, X. W., Yaqoob, T., \& Wang, J. X. 2010, Astrophys. J. Suppl. Ser., 187, 581

Smith, E. P., Heckman, T. M., Bothun, G. D., Romanishin, W. R., \& Balick, B. 1986, ApJ, 306, 64

Sulentic, J. W. 1989, ApJ, 343, 54

Sulentic, J. W., Marziani, P., \& Dultzin-Hacyan, D. 1999, Structure and Kinematics of Quasar Broad Line Regions, 175,175

Sulentic, J. W., Zwitter, T., Marziani, P., Dultzin-Hacyan D. 2000, ApJ, 545, 15

Sulentic, J. W., Zamfir, S., Marziani, P., Bachev, R., Calvani, M., Dultzin-Hacyan, D. 2003, ApJ, 597, 17

Sulentic, J. W., Repetto, P., Stirpe, G. M., et al. 2006, Astron. Astrophys., 456, 929

Sulentic, J. W., Marziani, P., 2015, arXiv:1506.01276

Sulentic, J. W., et al. 2015, in preparation

Yaqoob, T., George, I. M., Nandra, K., et al. 2001, Astrophys. J., 546, 759

Yaqoob, T., \& Padmanabhan, U. 2004, Astrophys. J., 604, 63

Vestergaard, M., \& Peterson, B. M. 2006, Astrophys. J., 641,689

Wandel, A., Peterson, B. M., \& Malkan, M. A. 1999, ApJ, 526,579

Welsh, W. F., Martino, D. L., Kawaguchi, G., \& Kollatschny, W. 2007, The Central Engine of Active Galactic Nuclei, 373, 29 
Wilson, A. S., Colbert, E. J. M. 1995, ApJ, 438, 62

Zamfir, S., Sulentic, J. W., Marziani, P., Dultzin, D. 2010 MNRAS, 403, 1759

Zheng, W., \& Sulentic, J. W. 1990, ApJ, 350, 512 